\title{
Métodos, instrumentos y procedimientos para conocer cómo se evalúan las competencias históricas $^{1}$
}

\section{Métodos, instrumentos e procedimentos para saber como as competências históricas são avaliadas}

\section{Methods, instruments and procedures to know how historical competences are evaluated}

\author{
Pedro Miralles* \\ José Monteagudo-Fernández ${ }^{*}$
}

\begin{abstract}
RESUMEN
Este artículo intenta explicar las características de la evaluación en la materia de Historia en lo referente a la tipología de preguntas, capacidades cognitivas, competencias históricas y tipos de contenidos que los exámenes y libros de texto demandan al alumnado de Educación Secundaria. Para ello se ha adoptado un diseño metodológico mixto, defendido en diversas investigaciones desde el área de didáctica de las ciencias sociales (BARCA, 2005; BARTON, 2005, 2012; GRANT, 2001; PRATS, 2002). Esta complementariedad de los enfoques cuantitativos y cualitativos creemos que nos permite acercarnos mejor a un fenómeno tan complejo como es la evaluación. Para el análisis cuantitativo se ha utilizado el paquete estadístico SPSS, en todo
\end{abstract}

1 Este trabajo forma parte del proyecto de investigación "La evaluación de las competencias y el desarrollo de capacidades cognitivas sobre Historia en Educación Secundaria Obligatoria" (EDU2015-65621-C3-2-R), subvencionado por el Ministerio de Economía y Competitividad de España y cofinanciado con fondos FEDER de la Unión Europea.

* Universidad de Murcia. Facultad de Educación. Campus Universitario de Espinardo. Espinardo, Murcia, España. E-mail: pedromir@um.es. https://orcid.org/0000-0002-9143-2145. E-mail: jose.monteagudo@um.es. https://orcid.org/0000-0003-2680-7622 
lo referente al estudio de frecuencias, medias, porcentajes, chi cuadrado, tablas de contingencia y análisis de la dependencia entre las variables. Por su parte, el tratamiento cualitativo se ha llevado a cabo a través de un análisis de cómo se ha construido la narrativa histórica en los exámenes y los libros de texto, a través de la frecuencia y repetición de hechos, conceptos, fechas y personajes. Además, hemos utilizado el programa Access para el procesamiento y vinculación de datos mediante la creación de tablas relacionales.

Palabras clave: Metodología. Evaluación. Pensamiento histórico. Educación Secundaria.

\title{
RESUMO
}

O artigo busca explicar as características de avaliação no ensino de história, tendo como referência os tipos de perguntas, habilidades cognitivas, habilidades e tipos de conteúdos históricos que os testes e livros didáticos exigem dos estudantes do ensino secundário. Para isso, adotamos uma concepção metodológica mista, defendida em várias investigações da área de ensino de ciências sociais (BARCA, 2005; BARTON, 2005, 2012; GRANT, 2001; PRATS, 2002). Acreditamos que a complementaridade das abordagens quantitativa e qualitativa permite a melhor aproximação de um fenômeno tão complexo como avaliação. Para a análise quantitativa foi utilizado SPSS, em todas as questões relativas ao estudo de frequências, médias, porcentagens, qui-quadrado, tabelas de contingência e análise da dependência entre as variáveis. De sua parte, o tratamento qualitativo foi realizado por meio da análise de como foi construída a narrativa histórica em testes e livros didáticos, através da frequência e repetição de fatos, conceitos, datas e personagens. Ademais, foi utilizado o programa Access para o processamento e ligação de dados através da criação de tabelas relacionais.

Palavras-chave: Metodologia. Avaliação. Pensamento histórico. Educação Secundária.

\begin{abstract}
This paper tries to explain the characteristics of the assessment in the subject of History in relation to the typology of questions, cognitive capacities, historical skills and types of contents that the exams and textbooks demand to the students of Secondary Education. To this end, a mixed methodological design has been adopted, defended in various investigations from the didactic area of the social sciences (BARCA, 2005; BARTON, 2005, 2012; GRANT, 2001; PRATS, 2002). We believe that this complementarity of the quantitative and qualitative approaches allows us to get closer to a phenomenon as complex as assessment. For the quantitative analysis the
\end{abstract}


statistical package SPSS has been used, in everything related to the study of frequencies, medians, percentages, chi-square, contingency tables and analysis of the dependence between the variables. On the other hand, the qualitative treatment has been carried out through an analysis of how the historical narrative has been constructed in exams and textbooks, through the frequency and repetition of facts, concepts, dates and characters. In addition, we have used the Access program for processing and linking data by creating relational tables.

Keywords: Methodology. Assessment. Historical thinking. Secondary Education.

\section{Presentación}

En el caso español, dos han sido los elementos que se han desarrollado con gran amplitud en los últimos años relacionados con el proceso de enseñanza-aprendizaje en general y con la historia en particular. Uno de ellos es el denominado pensamiento histórico, que ha conocido un gran progreso en los últimos decenios en países del ámbito anglosajón (BARTON; LEVSTIK, 2004; COOPER; CHAPMAN, 2009; LEE, 2005; LÉVESQUE, 2008; PECK; SEIXAS, 2008; SEIXAS; MORTON, 2013; VANSLEDRIGHT, 2011; WINEBURG, 2001), germánico (BORRIES, 1995; RÜSEN, 2005), hispanoamericano (HENRÍQUEZ; RUIZ, 2014; MORA; PAZ, 2012; PLÁ, 2012) y lusófono (BARCA, 2000; BARCA; SCHMIDT, 2013), pero no tanto en España, donde hasta la segunda década del nuevo milenio solamente podían destacarse las numerosas aportaciones de Carretero (2004, 2009, 2011) o Pagès (2009).

El otro componente ha sido la evaluación educativa, que ha adquirido también una gran importancia al calor de los programas internacionales de evaluación (PISA y otros) que pretenden medir los niveles de calidad de las instituciones y sistemas educativos para lograr la excelencia.

Partiendo de dicha realidad, este trabajo aborda los fundamentos metodológicos que el grupo de investigación Didáctica de las Ciencias Sociales (DICSO) de la Universidad de Murcia ha puesto en marcha para conocer y mejorar la enseñanza, el aprendizaje y la evaluación de la materia de Historia en la educación obligatoria teniendo como referente la teoría del pensamiento histórico y su reflejo en exámenes y libros de texto en la etapa de Educación Secundaria. 


\section{Sobre evaluación de competencias en Historia}

Siguiendo las tesis de Jorba y Sanmartí (1996), la evaluación es la emisión de un juicio de valor sobre algo que se quiere mejorar. Se trata de un proceso que consta de, al menos, tres momentos: recogida de información, análisis de dicha información y juicio sobre el resultado de este; y toma de decisiones de acuerdo con el juicio emitido.

Aunque tradicionalmente ha estado centrada en el aprendizaje del alumnado, desde una concepción actualizada la evaluación aparece como un instrumento al servicio del proceso de enseñanza y aprendizaje, integrada en el quehacer diario del aula y del centro educativo. Además, debe ser el punto de referencia en la adopción de decisiones que afectan a la intervención educativa, a la mejora del proceso y al establecimiento de medidas de refuerzo educativo o de adaptación curricular. Por ello, facilita el cambio educativo y el desarrollo profesional de los docentes, pues afecta no sólo a los procesos de aprendizaje de los alumnos y las alumnas, sino también a los procesos de enseñanza y a los proyectos curriculares.

El interés por la evaluación en España proviene de las reformas curriculares emprendidas en los últimos treinta años, que han puesto el foco de atención en la misma. Con la Ley Orgánica de Ordenación General del Sistema Educativo (LOGSE, 1990) la evaluación se situó como base del currículo del sistema educativo. La Ley Orgánica de Educación (LOE, 2006) supuso la entrada en juego de las competencias básicas y una evaluación más centrada en habilidades y procedimientos. La Ley Orgánica para la mejora de la calidad educativa (LOMCE, 2013) mantiene las competencias y ha introducido los estándares de aprendizaje, que pretenden determinar lo que el alumnado ha de aprender en cada asignatura (TREPAT, 2015), y las reválidas al final de etapa, que recientemente han sido eliminadas.

Para Coll y Martín (2006) han sido tres los motivos por los que la evaluación se ha convertido en el pilar que sustenta el mundo de la educación y su apartado legal. En primer lugar debido a su función reguladora, en la medida en que la información que proporciona la evaluación ayuda a tomar decisiones que corrigen y mejoran la educación. En segundo lugar, por su función de rendición de cuentas, es decir, el empleo de sus resultados de las evaluaciones como medidores de la consecución de los objetivos propuestos por un profesor o cualquier otra instancia del sistema educativo, y, finalmente, por el establecimiento de modelos de calidad de evaluación, traducidos en los niveles de rendimiento de que debe alcanzar el alumnado a una edad o nivel educativo concreto. Todo ello 
es lo que ha contribuido a colocar a la evaluación, concretamente la evaluación del rendimiento del alumnado, en el centro de las reformas curriculares que buscan una supuesta mejora de la educación.

Por tanto, la evaluación posee el papel de viga maestra sobre la que se sostiene todo cambio e innovación educativa; haciéndose imprescindible descubrir nuevas dimensiones dentro de la evaluación: nuevos criterios, procedimientos e instrumentos de evaluación (MIRALLES, 2015). A partir de ahora ésta no puede centrarse sólo en la evaluación de contenidos y en la utilización de unos instrumentos de evaluación determinados.

Si la evaluación se muestra como un elemento cardinal del proceso de enseñanza-aprendizaje, no es menos cierto que la disciplina histórica también cuenta con múltiples razones, como las aducidas por Prats y Santacana (2011), para justificar su presencia en los currículos educativos. En última instancia esta se explica por la necesidad de integrar de manera crítica al alumnado en la sociedad formando ciudadanía democrática (GALINDO, 2004).

Pero la formación de ciudadanía democrática que haga y se haga preguntas buscando respuestas a las mismas necesita de un proceso de instrucción en el aula que capacite al alumnado para ser crítico y autónomo. Ello pasa, a nuestro juicio, por el trabajo del pensamiento histórico en las clases de Historia.

El pensamiento histórico podría definirse como un metaconcepto que engloba habilidades relacionadas con la comprensión del pasado basadas en la interpretación de fuentes y la creación de narrativas históricas. Gómez y Miralles (2015) han realizado una síntesis de la evolución y el desarrollo del concepto de pensamiento histórico. La coexistencia de una tradición inglesa (LEE; ASHBY, 2000), que pone énfasis en los conceptos metodológicos con un perfil muy instrumental de la historia; y por otro lado la corriente alemana y la importancia de la conciencia histórica (RÜSEN,1992, 2015), que contempla la dimensión ética como finalidad fundamental de la historia, útil para la vida, línea ampliamente seguida en el ámbito lusófono (BARCA; SCHMIDT, 2013; SCHMIDT; GARCÍA, 2005), parece haber encontrado en las aportaciones canadienses y estadounidenses (LÉVESQUE, 2008, 2011; SEIXAS; MORTON, 2013; VANSLEDRIGHT, 2011, 2014; WINEBURG, 2001) una síntesis entre ambas. Dicha síntesis promueve la simulación del trabajo del historiador en el aula mediante la formulación de hipótesis sobre el pasado y su intento de demostrarlas a través de pruebas y de un método riguroso de análisis, poniendo en juego conceptos de primer orden (datos, fechas y hechos) y conceptos de segundo orden o metodológicos (DOMÍNGUEZ, 2015), relacionados con la relevancia histórica, el uso de fuentes históricas como pruebas, los cambios y las continuidades en la historia, las causas y consecuencias de los hechos históricos, la perspectiva histórica, e incorpora también la dimensión ética de la 
historia. Así se posibilita responder a cuestiones históricas y entender el pasado de forma compleja.

A través de la conjugación de los dos tipos de contenidos que integran el pensamiento histórico, el pasado se torna útil para comprender cómo se ha formado el presente, lo que favorece el conocimiento del contexto actual, hace posible su inclusión y dirige sus acciones hacia la mejora de la sociedad.

He aquí la razón por la que el conocimiento histórico y su evaluación es el núcleo central de la investigación que se muestra en estas páginas, entre otras razones porque articula en gran medida el desarrollo de las competencias sociales en la educación obligatoria.

Nos centramos en la evaluación del pensamiento histórico a través de los exámenes y los libros de texto porque los primeros son el principal instrumento para evaluar y calificar al alumnado (CALATAYUD, 2000; MERCHÁN, 2005, 2009; MONTEAGUDO; MIRALLES; VILLA, 2014). Por su parte, los libros de texto constituyen el recurso principal en la enseñanza de las ciencias sociales en general, y de la historia en particular; y porque la realización de las actividades de los libros de texto ocupan gran parte del tiempo dedicado a la enseñanza (PRATS, 2012; RODRÍGUEZ; GÓMEZ; SIMÓN, 2014; VALLS, 2008).

Tras contrastar en investigaciones anteriores (ALFAGEME; MIRALLES, 2014; GÓMEZ; MIRALLES, 2013, GÓMEZ; MONTEAGUDO; LÓPEZ-FACAL, 2012; MIRALLES; GÓMEZ; SÁNCHEZ, 2014; MIRALLES; GÓMEZ; MONTEAGUDO, 2012; MONTEAGUDO; MIRALLES; VILLA, 2014) que la práctica docente y evaluadora tradicional en la enseñanza social e histórica apenas se ha visto modificada por las sucesivas reformas educativas, consideramos que para alcanzar las finalidades que contempla la legislación educativa actual (formación de una ciudadanía consciente para participar en las nuevas sociedades europeas del siglo XXI) es necesario diseñar nuevas estrategias e instrumentos metodológicos y evaluativos para las aulas que lo hagan posible, al menos en mayor medida que hasta el momento.

\section{Diseño metodológico}

Pero para lograrlo, primero es necesario conocer la realidad de las aulas y, a partir de ahí, proponer actuaciones de mejora. Por ello el grupo de trabajo DICSO de la Universidad de Murcia, en coordinación con los grupos RODA de la Universidad de Santiago de Compostela (USC) y DHIGECS de la Universidad de Barcelona (UB), a través de los proyectos de investigación coordinados "La 
evaluación de las competencias básicas en Educación Secundaria Obligatoria desde las ciencias sociales" (EDU2012-37909-C03-03) y "La evaluación de las competencias y el desarrollo de capacidades cognitivas sobre Historia en Educación Secundaria" (EDU2015-65621-C3-2-R), financiados por el Ministerio de Economía y Competitividad, se propuso como objetivo principal, avanzar en la investigación sobre el desarrollo de competencias sociales entre estudiantes de Educación Secundaria Obligatoria (12-16 años) y su evaluación mediante el desarrollo de instrumentos específicos de enseñanza y aprendizaje de la historia que contribuyera a la formación escolar en valores democráticos, dando también respuesta a las demandas internacionales basadas en el paradigma de educar por competencias.

Partiendo de este objetivo general, los objetivos de investigación específicos que el grupo DICSO se propuso en ambos proyectos relativos al análisis de exámenes y libros de texto fueron los siguientes:

1. Analizar la tipología de ejercicios, contenidos y habilidades cognitivas sobre historia que se evaluaban a través de los exámenes;

2. Averiguar las habilidades de pensamiento y comprensión histórica del tiempo a través de los conceptos de primer y segundo orden, que se exigía conocer al alumnado en los exámenes, además del modelo de narrativa;

3. Estudiar los contenidos y las actividades formativas que se proponían en los libros de texto sobre los conocimientos históricos en Educación Secundaria Obligatoria y analizar la forma en que incorporan los conceptos de primer y segundo orden propios del pensamiento histórico, así como el modelo de narrativa que mostraban.

\section{Enfoque}

Las labores desarrolladas para alcanzar los objetivos propuestos tuvieron como base una investigación evaluativa, cuya finalidad última era la mejora u optimización del proceso de enseñanza-aprendizaje de la historia en Educación Secundaria Obligatoria; en concreto, se enmarcaba en un modelo de evaluación mixto u holístico en cuanto que integraba las perspectivas metodológicas cualitativa y cuantitativa y que permitió la realización de propuestas de mejora basadas en la recogida de información y de evidencias sistemática, rigurosa, objetiva, creíble, fiable y válida (LUKAS; SANTIAGO, 2009); desde este planteamiento se aunaba el potencial comprensivo-transformador de la investigación cualitativa 
con el valor explicativo-predictivo que aportaba la investigación cuantitativa (BISQUERRA; SABARIEGO, 2012).

En el marco de este enfoque evaluativo holístico, la perspectiva metodológica cualitativa que se asumió fue la fenomenológica, en cuanto que se trataba de describir, comparar, explicar y comprender la realidad de estudio desde la perspectiva de los participantes, en este caso los docentes, en el proceso de enseñanza-aprendizaje de la historia. Con la realización del estudio fenomenológico se intentó descubrir todos aquellos aspectos vinculados a los problemas de nuestra investigación que aparecían como pertinentes y significativos en las acciones de los actores socioeducativos siguiendo un procedimiento fundamentalmente inductivo adoptándolas pautas que recomiendan Sandín (2003) y Sabariego, Massot y Dorio (2012). Por actores socioeducativos no sólo contemplamos al profesorado de Historia de Educación Secundaria Obligatoria; también sus producciones (exámenes) y otros "actores" que proporcionaban información clave para la comprensión del contexto, del proceso y de los resultados que se fueron obteniendo (libros de texto). Como ya se ha apuntado, el procedimiento que fundamentalmente utilizan los estudios fenomenológicos como el que se planeó es el inductivo; de aquí que en el marco de este enfoque utilizáramos como estrategia analítica la teoría fundamentada (Grounded Theory) que nos permitió explicar las diferentes realidades generando teorías, modelos, conceptos, estándares... sobre la base de la recogida y análisis sistemático de los datos aportados por los actores mencionados (FLICK, 2007).

En el enfoque evaluativo holístico también se integra la perspectiva metodológica cuantitativa en cuanto que tratamos de identificar variables predictivas vinculadas a diferentes estrategias de enseñanza-aprendizaje que explicaran la evaluación del desarrollo del pensamiento histórico en el alumnado de Educación Secundaria Obligatoria; ello lo realizamos utilizando un diseño cuasi experimental en los contextos naturales de las aulas de los centros de esta etapa educativa de la Región de Murcia (RAMOS, 2011). No obstante, el logro de otros objetivos de esta investigación también exigía que adoptáramos un diseño descriptivo-correlacional que nos permitiera identificar factores, compararlos y correlacionarlos para que estén en la base de la explicación de las realidades objeto de estudio (MARTÍN, 2011).

El empleo de la mencionada metodología de investigación de integración cualitativa-cuantitativa vino motivada por nuestra pretensión de dar a nuestra investigación un cariz multiparadigmático siguiendo la recomendación de Del Rincón, Arnal, Latorre y Sans de que: "En Ciencias Sociales la diversidad metodológica posibilita el estudio de la realidad social desde diversas ópticas, ya que ninguna perspectiva metodológica por si sola responde totalmente a las preguntas que pueden formularse en el contexto social" (1995, p. 26). 
La finalidad era alcanzar una mayor comprensión del problema que se nos presentaba, enriqueciendo y ampliando de esta manera el conocimiento que surgiera de nuestro estudio, generando distintas propuestas y estrategias de acción. Como sostuvieron Evertson y Green (1989), la combinación de metodologías en indagaciones en ciencias sociales es una de las tácticas más válidas para la ampliación de las nociones acerca de fenómenos educativos.

\section{Contexto y participantes}

El contexto en el que se desarrolló la investigación fue la introducción de la nueva ley educativa (LOMCE) en España. El problema de estudio de nuestros proyectos y los objetivos a los que se pretendíamos responder afectaban al proceso de enseñanza-aprendizaje de la Historia en la etapa de la Educación Secundaria Obligatoria. Este planteamiento contextual inicial, así como el enfoque de nuestra investigación (holístico), eran imprescindibles para definir quiénes y cuáles eran los actores y participantes de la misma de los que íbamos a recoger la información necesaria para el logro de los objetivos. Se han empleado los métodos no probabilísticos (SABARIEGO, 2012), en particular:

- Los libros de texto que se utilizaban para la enseñanza de la historia en la etapa de Educación Secundaria Obligatoria. Se requirió del análisis de estas unidades muestrales para estudiar los contenidos y las actividades formativas que se proponían y conocer la forma en que incorporaban las capacidades cognitivas y los contenidos propios del pensamiento histórico, así como la tipología de ejercicios y el modelo de narrativa. Fueron seleccionados los libros de texto de algunas de las editoriales (Vicens Vives, Santillana, SM...) más utilizadas por los profesores de ESO en la Región de Murcia (GÓMEZ, 2014).

- Los exámenes de Historia realizados por el alumnado para conocer los conocimientos asociados al pensamiento histórico, modelo de narrativa, tipología de ejercicios y capacidades cognitivas que éstos exigían al alumnado y contrastarlo con lo propuesto en las competencias. $\mathrm{La}$ selección de estos exámenes se hizo tomando como conglomerados los grupos-clase y utilizando un procedimiento de muestreo no probabilístico por cuotas en el que se fijó una muestra representativa atendiendo a la titularidad del centro, ubicación del centro (región y comarca) y centro bilingüe/no bilingüe. 
En la tabla 1 puede verse la relación por curso del total de 157 exámenes y 18 libros de texto analizados, de los que se obtuvo un total de 1427 ejercicios de examen y 943 actividades de libro de texto.

TABLA 1. MUESTRA POR CURSO ACADÉMICO DE LIBROS DE TEXTO Y EXÁMENES

\begin{tabular}{|c|c|c|}
\hline Nivel escolar & $\begin{array}{c}\text { Libros de texto }\left(\text { n. }^{\circ} \text { de }\right. \\
\text { actividades) }\end{array}$ & $\begin{array}{c}\text { Exámenes (n. }{ }^{\circ} \text { de } \\
\text { ejercicios) }\end{array}$ \\
\hline $1 .^{\circ} \mathrm{ESO}$ & $6(102)$ & $49(493)$ \\
\hline $2 .^{\circ} \mathrm{ESO}$ & $9(688)$ & $73(635)$ \\
\hline $4 .^{\circ} \mathrm{ESO}$ & $3(153)$ & $35(299)$ \\
\hline Total & $18(943)$ & $157(1427)$ \\
\hline
\end{tabular}

FUENTE: Elaboración propia

\section{Recogida de información: instrumentos y procedimientos}

La recogida de información se realizó mediante técnicas e instrumentos cualitativos interactivos y no interactivos y técnicas e instrumentos cuantitativos; todos ellos diseñados por el equipo de investigación para dar respuesta a los objetivos del trabajo; de aquí que los instrumentos que se elaboraron fueron necesariamente sometidos a un proceso previo de validación antes de su utilización con los participantes, mediante un estudio piloto previo o mediante técnicas tales como la triangulación de fuentes, fiabilidad interjueces mediante la W de Kendall, análisis factorial exploratorio con rotación Varimax y criterio de extracción de factores de Kaisser, análisis correlacionales...). En concreto, los instrumentos que se construyeron y utilizaron fueron protocolos para el registro sistemático de la información que se observó del análisis de los libros de texto utilizados para la enseñanza de la historia, así como de los exámenes, actividades y ejercicios evaluativos realizados por el alumnado de Educación Secundaria Obligatoria.

La recogida de información fue realizada por los miembros del grupo de investigación que compartían un mismo marco de referencia conceptual-teórico y experiencial, formativo y práctico, en torno a la problemática de estudio y a los procedimientos de aplicación de las técnicas e instrumentos; de este modo, la influencia de variables extrañas asociadas a la variabilidad de la intervención externa fue objetivada en gran medida. 


\section{Plan de tratamiento y análisis de la información}

El enfoque holístico de la investigación demandaba la complementariedad de estrategias analíticas cualitativas y cuantitativas, en función de la naturaleza de la información recogida.

La información cualitativa fue analizada mediante el programa ATLAS. ti (versión 6.2), según un planteamiento fundamentalmente inductivo siguiendo el método de la teoría fundamentada (GroundedTheory), coherente con la propuesta de Miles y Huberman (1994) adaptada por Serrano Pastor (1999). Se siguieron estas etapas:

a) La información textual, gráfica y multimedia se recogió en ficheros que constituyeron los documentos primarios de las unidades hermenéuticas creadas según la temática u objetivo de estudio;

b) Según un criterio temático y atendiendo a diferentes variantes discursivas relevantes en nuestra investigación se definieron las unidades de análisis o citas de la información textual, gráfica y multimedia, extrayendo las categorías y los códigos según una estrategia inductiva y mixta;

c) Se agruparon las categorías y los códigos en familias, originando metacategorías y supracategorías que estuvieran en la base de la descripción, explicación y comprensión de las temáticas de estudio;

d) Se elaboraron redes semánticas que reflejasen las teorías, los modelos o conceptos emergentes sobre la problemática de estudio.

Los datos cuantitativos medidos en diferentes escalas de medida (nominal, ordinal, de intervalos y de razón o de proporción) y los diseños de investigación (descriptivo y cuasiexperimental) fueron tratados y analizados con el programa estadístico IBM SPSS (v. 20), siguiendo las siguientes etapas una vez elaboradas las correspondientes matrices de datos:

a) Análisis exploratorio de la información para detectar los valores fuera de rango y analizar la distribución de los datos con el fin de determinar la realización de las técnicas estadísticas más adecuadas (técnicas paramétricas o no paramétricas). Para ello se calcularon estadísticos descriptivos y los gráficos exploratorios box-plotystem-and-leaf;

b) Análisis confirmatorio descriptivo. Desde un planteamiento univariado, bivariado y multivariado se calcularon los estadísticos descriptivos de las variables implicadas, así como pruebas de asociación y pruebas de correlación en función de la naturaleza de las variables intervinientes; 
c) Análisis confirmatorio inferencial. Se realizaron pruebas de comparación de dos medias (o k medias) para muestras independientes y dependientes, utilizando como variables de agrupamiento o factores género, curso, titularidad de centro, ubicación, centro bilingüe/no bilingüe, experiencia docente y otras de carácter predictivo;

d) Análisis de regresión múltiple que trataba de explicar la relación y variabilidad existente entre las variables independientes y la evaluación del pensamiento histórico desde la perspectiva de los docentes.

En todas las pruebas estadísticas que implicaban contraste de hipótesis se asumió un nivel crítico $(\mathrm{p}<=.05)$ como es propio en las investigaciones en el ámbito educativo y de las ciencias sociales.

\section{Conclusiones de la metodología y los instrumentos utilizados}

El objetivo del presente trabajo era el de mostrar el diseño metodológico seguido por el grupo de investigación DICSO para conocer la naturaleza de las competencias sociales evaluadas entre estudiantes de Educación Secundaria Obligatoria (12-16 años) mediante los conceptos de la teoría del pensamiento histórico en exámenes y libros de texto.

Las decisiones metodológicas tomadas sobre el diseño de investigación y los instrumentos empleados, teniendo en cuenta los principios teóricos que las fundamentan, creemos que han permitido acercarnos a la realidad de lo que ocurre en las aulas, pues se ha seguido un riguroso proceso de análisis a pesar de su complejidad; un proceso que puede facilitar a otros equipos investigadores similares las tareas que tengan que establecer en sus propios trabajos.

El enfoque evaluativo holístico empleado, integrando las perspectivas cuantitativa y cualitativa, ha posibilitado aproximarnos de manera más exacta y comprensiva al proceso de enseñanza y evaluación que lleva a cabo el profesorado de ESO en la asignatura de Historia. Formar una ciudadanía autónoma, crítica y activa requiere superar la enseñanza memorística tradicional y enseñar métodos de investigación propios de historiador que lleve al alumnado a lanzar hipótesis, investigar, comprobar y contrastar la información obtenida, y actuar en consecuencia. Trabajar las competencias del pensamiento histórico abunda en este camino.

Consideramos que hemos justificado las razones por las que hemos elegido, dentro del método cuantitativo de investigación, la codificación de las categorías 
que así lo permitieron en el paquete estadístico SPSS para conocer qué tipo de contenidos, capacidades cognitivas y competencias recogidas en la teoría del pensamiento histórico se evaluaban en los exámenes de Historia.

Del mismo modo, creemos que hemos acreditado los motivos por los que optamos por el tratamiento cualitativo de los datos a través de un análisis de cómo se ha construido la narrativa histórica nacional en los exámenes y los libros de texto, a través de la frecuencia y repetición de hechos, conceptos, fechas y personajes.

Juzgamos adecuados los criterios seguidos para la selección de los participantes en la investigación con la finalidad de mostrar una imagen lo más acertada posible de la evaluación y aprendizaje de las capacidades cognitivas y los conceptos de pensamiento histórico, y su relación con las competencias.

Se ha especificado también el proceso de análisis seguido, que se basa en la ya mencionadacombinación de un enfoque cuantitativo y otro cualitativo, que estimamos conveniente debido a su utilidad para aportar información más cercana a la realidad de lo que acontece en el día a día de las aulas y mejorar los procesos de enseñanza-aprendizaje. Por otro lado, se trata de una labor enriquecedora también para los investigadores porque el trabajo se realiza en equipos interdisciplinares en los que se producen constantes interacciones que potencian el aprendizaje y la colaboración.

\section{REFERENCIAS}

ALFAGEME, M. B.; MIRALLES, P. El profesorado de Geografía e Historia de Enseñanza Secundaria ante la evaluación. Educar em Revista, v. 30, n. 52, p. 193-209, 2014.

BARCA, I. O pensamento histórico dos jovens. Braga: Universidade do Minho, 2000.

BARCA, I. Till new facts are discovered': Students' ideas about objectivity in History. In: ASHBY, R.; GORDON, P.; LEE, P. (eds.). International Review of History Education. Understanding History: Recent research in History education. New York: Routledge Falmer, 2005. p. 68-82.

BARCA, I.; SCHMIDT, M. A. La consciencia histórica de los jóvenes brasileños y portugueses y su relación con la creación de identidades nacionales. Educatio Siglo XXI, v. 31, n. 1, p. 25-46, 2013.

BARTON, K. Best not to Forget Them': Adolescents' judgments of historical significance in Northern Ireland. Theory and Research in Social Education, n. 33, p. 9-44, 2005. 
BARTON, K. Applied Research: Educational research as a way of seeing. In: MCCULLY, A.; MILLS, G.; VAN BOXTEL, C. (Eds.). The Professional Teaching of History: UK and Dutch perspectives. Coleraine: History Teacher Education Network, 2012. p. 1-15.

BARTON, K.; LEVSTIK, L. Teaching History for the Common Good. New Jersey: Lawrence Erlbaum, 2004.

BISQUERRA, R.; SABARIEGO, M. El proceso de investigación (parte I). In: BISQUERRA, R. (Coord.). Metodología de la investigación educativa. Madrid: La Muralla, 2012. p. 89-125.

BORRIES, B. V. Exploring the Construction of Historical Meaning: Cross-Cultural Studies of Historical Consciousness Among Adolescents. In: BOS, W.; LEHMANN, R. H. (Eds.). Reflections on Educational Achievement. Munster/New York, 1995. p. 25-49.

CALATAYUD, M. A. La supremacía del examen: la evaluación como examen. Su uso y abuso, aún, en la educación primaria. Bordón. Revista de Pedagogía, v. 52, n. 2, p. 165-178, 2000.

CARRETERO, M. Comprensión y aprendizaje de la historia. In: PRATS, J. et al. Enseñanza y aprendizaje de la historia en la educación básica. México DF: Secretaría de Educación Pública, Gobierno de México, 2011, p. 69-104.

CARRETERO, M.; VOSS, J. (Comps.). Aprender y pensar la historia. Buenos Aires: Amorrortu, 2004.

CARRETERO, M.; LÓPEZ, C. Estudios cognitivos sobre el conocimiento histórico: aportaciones para la enseñanza y la alfabetización histórica. Enseñanza de las Ciencias Sociales, n. 9, p. 79-83, 2009.

COLL, C.; MARTÍN, E. Vigencia del debate curricular. Aprendizajes básicos, competencias y estándares. Segunda Reunión del Comité Intergubernamental del PRELAC, Santiago de Chile. 2006.

COOPER, H.; CHAPMAN, A. (Eds.). Constructing History, 11-19. London: Sage, 2009.

DEL RINCÓN, D.; ARNAL, J.; LATORRE, A.; SANS, A. Técnicas de investigación en Ciencias Sociales. Madrid: Dykinson, 1995.

DOMÍNGUEZ, J. Pensamiento histórico y evaluación de competencias. Barcelona: Graó, 2015.

EVERTSON, C. M.; GREEN, J. L. La índole de la observación y de los instrumentos observacionales. In: WITTROCK, M. C. La investigación de la enseñanza, II. Métodos cualitativos y de observación. Barcelona: Paidós, 1989. p. 306-310.

FLICK, U. Introducción a la investigación cualitativa. Madrid: Morata, 2007.

GALINDO, R. Otra enseñanza de las ciencias sociales es posible. In: VERA, M. I.; PÉREZ, D. (Coords.). Formación de la ciudadanía: las TICs y los nuevos problemas. Alicante: Universidad de Alicante, 2004. p. 1-13. 
GÓMEZ, C. J. Pensamiento histórico y contenidos disciplinares en los libros de texto. Un análisis exploratorio de la Edad Moderna en $2^{\circ}$ de la ESO. Ensayos. Revista de la Facultad de Educación de Albacete, v. 9, n. 1, 2014.

GÓMEZ, C. J.; MONTEAGUDO, J.; LÓPEZ, R. El examen y la evaluación de los contenidos de ciencias sociales en tercer ciclo de Educación Primaria. Capacidades, conceptos y procedimientos. Revista Electrónica Interuniversitaria de Formación del Profesorado, v. 15, n. 1, p. 37-49, 2012.

GÓMEZ, C. J.; MIRALLES, P. Los contenidos de ciencias sociales y las capacidades cognitivas en los exámenes de tercer ciclo de Educación Primaria ¿Una evaluación en competencias? Revista Complutense de Educación, v. 24, p. 91-121, 2013.

GÓMEZ, C. J.; MIRALLES, P. ¿Pensar históricamente o memorizar el pasado? La evaluación de los contenidos históricos en la educación obligatoria en España. Revista de Estudios Sociales, v. 52, p. 52-68, 2015. doi:10.7440/res52.2015.04

GRANT, S. G. It's just the Facts, or is it? The relationship between teachers' practices and students' understandings of History. Theory and Research in Social Education, v. 29, n. 1, p. 65-108, 2001.

HENRÍQUEZ, R.; RUIZ, M. Chilean students learn to think historically: construction of historical causation through the use of evidence in writing. Linguistics and Education, v. 25 , p. $145-167,2014$.

JORBA, J.; SANMARTÍ, N. Enseñar, aprender y evaluar: un proceso de evaluación continua: propuestas didácticas para las áreas de ciencias de la naturaleza y matemáticas. Madrid: MEC, 1996.

LEE, P. Putting principles intro practice: understanding history. In: DONOVAN, M.; BRANSFORD, J. (Eds.). How students learn: History in the classroom. Washington: National Academies Press, 2005. p. 31-77.

LEE, P.; ASHBY, R. Progression in Historical Understanding among Students Ages 7-14. In: STEARNS, P. N.; SEIXAS, P.; WINEBURG, S. (Eds.). Knowing, Teaching and Learning History. National and international perspectives. New York/London: New York University Press, 2000. p. 199-222.

LÉVESQUE, S. Thinking Historically. Educating students for the 21th Century. Toronto: University of Toronto Press, 2008.

LÉVESQUE, S. What it Means to Think Historically? In: CLARK, P. (Ed.). New Possibilities for the Past. Shaping history education in Canada. Vancouver/Toronto: UBC Press, 2011. p. 115-138.

LUKAS, J. F.; SANTIAGO, K. Evaluación educativa. Madrid: Alianza Editorial, 2009.

MARTÍN, B. Investigación descriptiva. In: CUBO, S.; MARTÍN, B.; RAMOS, J. L. Métodos de investigación y análisis de datos en ciencias sociales y de la salud. Madrid: Pirámide, 2011. p. 373-385. 
MERCHÁN, F. J. Enseñanza, examen y control: profesores y alumnos en la clase de Historia. Barcelona: Octaedro, 2005.

MERCHÁN, F. J. Hacer extraño lo habitual. Microsociología del examen en la clase de Historia. Íber. Didáctica de las Ciencias Sociales, Geografia e Historia, v. 1, n. 60, p. 21-34, 2009.

MILES, M. B.; HUBERMAN, A. M. Qualitative Data Analysis: An expanded sourcebook. Thounsand Oaks, CA: Sage Publications, 1994.

MIRALLES, P. Investigaciones sobre evaluación en ciencias sociales en la educación básica. Propuestas para la mejora de la práctica evaluativa. In: MIRALLES, P. Didáctica de la historia. Problemas y métodos. México: Editorial y Servicios Culturales El Dragón Rojo, 2015. p. 99-129.

MIRALLES, P.; GÓMEZ, C.; SÁNCHEZ, R. Dime qué preguntas y te diré qué evalúas y enseñas. Análisis de los exámenes de Ciencias Sociales en tercer ciclo de Educación Primaria. Aula Abierta, v. 42, n. 2, p. 83-89, 2014.

MIRALlES, P.; GÓMEZ, C.; MONTEAGUDO, J. La evaluación de la competencia social y ciudadana en ciencias sociales al finalizar las etapas de Primaria y Secundaria. Investigación en la Escuela, v.78, p.19-30, 2012.

MONTEAGUDO, J.; MIRALLES, P.; VILLA, J. L. Evaluación de la materia de Historia en Secundaria. El caso de la Región de Murcia. Saarbrücken: Publicia, 2014.

MORA, G.; PAZ, R. El modelo de educación histórica. Experiencia de innovación en la educación básica de México. Enseñanza de las Ciencias Sociales. Revista de Investigación, n. 11, p. 87-98, 2012.

PAGÈS, J. El desarrollo del pensamiento histórico como requisito para la formación democrática de la ciudadanía. Reseñas de Enseñanza de la Historia, n. 7, p. 69-91, 2009.

PECK, C.; SEIXAS, P. Benchmarks of Historical Thinking: First Steps. Canadian Journal of Education, v. 31, n. 4, p. 1015-1038, 2008.

PLÁ, S. La enseñanza de la historia como objeto de investigación. Secuencia, n. 84, p. 163-184, 2012.

PRATS, J. Hacia una definición de la investigación en didáctica de las Ciencias Sociales. Enseñanza de las Ciencias Sociales. Revista de Investigación, n. 1, p. 81-89, 2002.

PRATS, J. Criterios para la elección del libro de texto de Historia. Íber. Didáctica de las Ciencias Sociales, Geografia e Historia, v. 2, n. 70, p. 7-13, 2012.

PRATS, J.; SANTACANA, J. Por qué y para qué enseñar Historia. In: PRATS, J. (Coord.). Didáctica de la Geografía y la Historia. Barcelona: Graó, 2011. p. 13-30.

RAMOS, J. L. Investigación cuasi experimental. In: CUBO, S.; MARTÍN, B.; RAMOS, J. L. Métodos de investigación y análisis de datos en ciencias sociales y de la salud. Madrid: Pirámide, 2011. p. 329-371. 
RODRÍGUEZ, R.; GÓMEZ, C. J.; SIMÓN M. M. Conocimiento y pensamiento histórico en los manuales escolares de tercer ciclo de Primaria. Un análisis comparativo. In: NORTES, R.; ALONSO, J. I. (Eds.). Investigación educativa en Educación Primaria. Murcia: Editum, 2014. p. 369-380.

RÜSEN, J. El desarrollo de la competencia narrativa en el aprendizaje histórico. Una hipótesis ontogenética relativa a la conciencia moral. Propuesta Educativa, n. 7, p. 27-36, 1992.

RÜSEN, J. History: Narration, Interpretation, Orientation. New York: Berghahn, 2005. RÜSEN, J. Teoria da história: uma teoria da história como ciência. Curitiba: Editora UFPR, 2015.

SABARIEGO, M. La investigación educativa. Génesis, evolución y características. In: BISQUERRA, R. (Coord.). Metodología de la investigación educativa. Madrid: La Muralla, 2012. p. 57-87.

SABARIEGO, M.; MASSOT, I.; DORIO, I. Métodos de investigación cualitativa. In: BISQUERRA, R. (Coord.). Metodología de la investigación educativa. Madrid: La Muralla, 2012. p. 293-328.

SANDIN, M. Investigación cualitativa en educación. Fundamentos y tradiciones. Barcelona: McGraw Hill, 2003.

SCHMIDT, M. A.; GARCIA, T. M. A formação da consciência histórica de alunos e professores e o cotidiano em aulas de História. Cad. Cedes, Campinas, v. 25, n. 67, p. 297-308, set./dez. 2005.

SEIXAS, P.; MORTON, T. The Big Six Historical Thinking Concepts. Toronto: Nelson, 2013.

SERRANO PASTOR, F. J. Análisis de relatos. In: SÁEZ, J.; ESCARBAJAL, A.; GARCÍA, A.; CAMPILLO, M. (Coords.). Cuentos pedagógicos, relatos educativos. Murcia: Diego Marín, 1999. p. 33-47.

TREPAT, C. La Historia en la LOMCE. ESO y Bachillerato. Íber. Didáctica de las Ciencias Sociales, Geografia e Historia, v. 1, n. 79, p. 49-59, 2015.

VALLS, R. Los textos escolares de Historia: una propuesta de análisis y valoración. In: PRATS, J.; ALBERT, J. M. (Eds.). Ellsllibres de text i l'ensenyament de la Història, Barcelona: Universitat de Barcelona, 2008. p. 63-72.

VANSLEDRIGHT, B. The Challenge of Rethinking History Education. On practice, theories, and policy. New York: Routledge, 2011.

VANSLEDRIGHT, B. Assessing Historical Thinking \& Understanding. Innovate designs for new standards. New York: Routledge, 2014. 
MIRALLES, P.; MONTEAGUDO-FERNÁNDEZ, J. Métodos, instrumentos y procedimientos para...

WINEBURG, S. Historical Thinking and Other Unnaturals Acts: Charting the future of teaching the past. Philadelphia: Temple University Press, 2001.

Texto recibido el 18 de enero de 2019.

Texto aprobado el 23 de enero de 2019. 